\title{
DCT4 - a new member of the dicarboxylate transporter family in $\mathrm{C}_{4}$ grasses
}

\author{
Sarit Weissmann ${ }^{1}$, Pu Huang ${ }^{1+}$, Koki Furuyama ${ }^{2}$, Madeline A. Wiechert ${ }^{1}$, Mitsutaka Taniguchi ${ }^{2}$, James C. \\ Schnable ${ }^{3^{*}}$, Thomas P. Brutnell ${ }^{4}$ and Todd C. Mockler ${ }^{1^{*}}$ \\ ${ }^{1}$ Donald Danforth Plant Science Center, St. Louis, MO, 63132, USA. \\ ${ }^{2}$ Graduate School of Bioagricultural Sciences, Nagoya University, Nagoya, Aichi, Japan. \\ ${ }^{3}$ Computational Sciences Initiative, Center for Plant Science Innovation, Department of Agronomy and Horticulture, University \\ of Nebraska-Lincoln, Lincoln, NE, USA. \\ ${ }^{4}$ Chinese Academy of Agricultural Sciences, Biotechnology Research Institute, Beijing, China. \\ ${ }^{+}$Current Address: BASF Corporation, 26 Davis Dr., Durham, NC 27709, USA. \\ ${ }^{*}$ Corresponding author
}

\begin{abstract}
Malate transport shuttles atmospheric carbon into the Calvin-Benson cycle during NADP-ME $\mathrm{C}_{4}$ photosynthesis. Previous characterizations of several plant dicarboxylate transporters (DCT) showed that they efficiently exchange malate across membranes. Here we identify and characterize a previously unknown member of the DCT family, DCT4, in Sorghum bicolor. We show that SbDCT4 exchanges malate across membranes and its expression pattern is consistent with a role in malate transport during $\mathrm{C}_{4}$ photosynthesis. SbDCT4 is not syntenic to the characterized photosynthetic gene ZmDCT2, and an ortholog is not detectable in the maize reference genome. We found that the expression patterns of $D C T$ family genes in the leaves of Z. mays, and S. bicolor varied by cell type. Our results suggest that subfunctionalization of members of the $D C T$ family for the transport of malate into the bundle sheath (BS) plastids occurred during the process of independent recurrent evolution of $C_{4}$ photosynthesis in grasses of the PACMAD clade. This study confirms the value of using both syntenic information and gene expression profiles to assign orthology in evolutionarily related genomes.
\end{abstract}




\section{INTRODUCTION}

Three subtypes of $C_{4}$ photosynthesis are generally recognized as defined by the primary decarboxylase in the bundle sheath (BS) cells: chloroplastic NADP-dependent malic enzyme (NADP-ME); mitochondrial NAD-dependent malic enzyme (NAD-ME); and cytosolic phosphoenolpyruvate carboxykinase (PEPCK) (Hatch and Slack, 1966; Hatch, 1971; Rathnam and Edwards, 1977). Different plant species may contain various combinations of these three subtypes (Hatch, 1971; Chapman and Hatch, 1979; Furbank, 2011; Pick et al., 2011; Wang et al., 2014b). The movement and exchange of malate across membranes, by dicarboxylate transporters (DCTs/DiTs), plays a significant role during photosynthesis in NADP-ME and NAD-ME $C_{4}$ species (Ding et al., 2015). In $C_{3}$ plants, DCTs are crucial to nitrate assimilation, such as the GS/GOGAT cycle and photorespiration (Linka and Weber, 2010; Kinoshita et al., 2011). Taniguchi et al. characterized several plant DCTs that efficiently exchange malate across membranes (Taniguchi et al., 2002; Taniguchi et al., 2004). The differential expression of $C_{4}$ photosynthesis genes in mesophyll (M) and BS cells (John et al., 2014; Tausta et al., 2014; Wang et al., 2014a) suggests that different malate transporters may be needed to move malate out of the chloroplasts of $\mathrm{M}$ cells and into the chloroplasts of BS cells. In Zea mays, an NADP-ME $\mathrm{C}_{4}$ grass, dicarboxylate transporter-2 (ZmDCT2, GRMZM2G086258) moves malate into the chloroplast of BS cells during $C_{4}$ photosynthesis (Ding et al., 2015). ZmDCT2 plays a critical role during $C_{4}$ photosynthesis in Z. mays, and its absence severely impairs plant growth and development (Ding et al., 2015). The role of DCTs in $C_{4}$ photosynthesis in other species, however, remains unknown.

Z. mays is the best characterized and functionally annotated $C_{4}$ grass species. As such, it is a useful reference for identification of photosynthesis-related genes in poorly characterized $\mathrm{C}_{4}$ grasses and for resolving orthology (John et al., 2014; Ding et al., 2015; Huang et al., 2017). Microsynteny, the comparison of collinearity among related species, is a reliable approach to determine orthology and predict the function of a gene (Bennetzen and Freeling, 1997; Chen et al., 1997; Tikhonov et al., 1999; Bennetzen, 2000; Kumar et al., 2009; Jin et al., 2016). Davidson et al. (Davidson et al., 2012) showed that syntenic orthologs are likely to have conserved functions and expression patterns across lineages. Here we identify a new member of the DCT family, DCT4, which is not syntenic to the photosynthetic gene $Z m D C T 2$ and is not detected in the maize reference genome. We demonstrate that Sorghum bicolor DCT4 (SbDCT4) efficiently exchanges malate across membranes, consistent with a malate transport role in $\mathrm{C}_{4}$ photosynthesis. We characterize the diverse expression patterns of $D C T$ genes in leaves of multiple 
grass species. We also propose that sub-functionalization of DCTs in grasses of the PACMAD clade (Sanchez-Ken and Clark, 2010) occurred during independent recurrent evolution of $\mathrm{C}_{4}$ photosynthesis.

\section{RESULTS}

\section{Identification of DCT4 in Sorghum bicolor}

To learn more about $\mathrm{C}_{4}$-related dicarboxylate transporters in species evolutionarily related to maize, we identified the syntenic ortholog of ZmDCT2 in S. bicolor. Two genes, Sobic.007G226700 and Sobic.007G226800, are present at the predicted syntenic orthologous position on chromosome 7. We refer to them as SbDCT2.1 and SbDCT2.2, respectively (Figure 1). ZmDCT2 is abundantly expressed (Table 1), and its expression is enriched in BS cells of maize leaves (Figure 2) (Li et al., 2010; Tausta et al., 2014; Ding et al., 2015). In contrast, the expression profiles of SbDCT2.1 and SbDCT2.2 in S. bicolor leaves are low (Table 1). SbDCT2.1 expression is slightly enriched in the M cells whereas SbDCT2.2 is enriched in BS cells (Figure 2). We also analyzed the transcript levels of two other S. bicolor dicarboxylate transporters, SbDCT1 (Sobic.002G233700) and SbOMT1 (Sobic.008G112300). These genes are the orthologs of the Z. mays genes ZmDCT1 (GRMZM2G040933) and Zm-oxoglutarate/malate transporter 1 (ZmOMT1; GRMZM2G383088), respectively. We found that SbDCT1 expression, similar to that of $Z m D C T 1$, is relatively low (Table 1), and only slightly differentially expressed in $\mathrm{M}$ cells relative to BS cells (Figure 2). The expression of both ZMOMT1 and SbOMT1 is relatively high (Table 1), and both are slightly enriched in $\mathrm{M}$ cells (Figure 2).

In $\mathrm{C}_{4}$ species, the expression of many photosynthetic genes is enriched in either BS and $\mathrm{M}$ cells (Li et al., 2010; John et al., 2014; Tausta et al., 2014; Weissmann et al., 2015; Rao et al., 2016). In NADP-ME species, two transporters, one within the BS cells and another in M cells, move malate in and out of the chloroplast during $\mathrm{C}_{4}$ photosynthesis (Brautigam et al., 2008; Weissmann and Brutnell, 2012; John et al., 2014; Tausta et al., 2014; Wang et al., 2014a). However, in sorghum leaves, we found only one highly expressed dicarboxylate transporter, SBOMT1, that showed slightly enriched expression in M cells. Therefore we screened the sorghum genome for additional homologs of known maize DCTs. We identified the gene Sobic.004G035500 that showed homology to ZmDCT1 and ZmDCT2 but was not syntenic to either gene (Figure 1). DCT3 is the name of the second transcript of ZmDCT2 (Taniguchi et al., 2004), so we named this new gene SbDCT4. No syntenic ortholog of SbDCT4 is present in the reference genomes of Z. mays or Oryza sativa. The absence of syntenic conservation between S. bicolor and Z. mays and the lack of direct orthologs in Z. mays or $C_{3}$ species prevented identification of DCT4 in a 
previous bioinformatic screen for $C_{4}$ photosynthesis genes (Huang et al., 2017). The expression of SbDCT4 is moderately abundant (Table 1) and strongly enriched in the BS cells of S. bicolor leaves (Figure 2).

\section{SbDCT4 is an efficient malate transporter}

To verify the ability of SbDCT4 to transport malate, we cloned coding sequences from the three sorghum $D C T$ genes, SBDCT1, SbDCT2, and SbDCT4. We measured the malate transport activities of the recombinant proteins expressed in yeast. SbDCT4 was an efficient malate transporter (Table 2). The $K_{\mathrm{m}}$ of SbDCT4 was similar to that of SbDCT1, and the affinity for malate was highest in SbDCT2 among the three SbDCTs (Table 2), consistent with the relative malate transport activities reported for maize DCT1 and DCT2 (Taniguchi et al., 2004).

\section{Phylogenetic distribution of $D C T$ genes in grasses}

To understand the relationship of SbDCT4 to other grass DCT genes, we searched the genomes of the grass species Setaria italica, Urochloa fusca, Brachypodium distachyon, and Dichanthelium oligosanthes. In S. italica, an NADP-ME $\mathrm{C}_{4}$ species, we identified a dicarboxylate transporter, Seita.9G375100, that showed no syntenic orthologous relationship with dicarboxylate transporter genes in other available grass genomes. Phylogenetic analysis showed that this gene clustered with SbDCT4 but not with SbDCT1 and SBDCT2 (Figure 3). We designated this gene SiDCT4. We did not detect orthologs, syntenic or otherwise, in $U$. fusca, a PEPCK $C_{4}$ species, or in the two $C_{3}$ species. To expand the search for DCT4 in other grasses currently lacking genome assemblies, we examined leaf-derived transcript assemblies for Aristida congesta, Eriachne aristidea, Chasmanthium laxum, Danthoniopsis dinteri, Anthephora pubensis, Echinochloa esculenta, Paspalum vaginatum, and Arundinella hirta (Huang et al., in preparation). We then used the predicted coding sequences of the DCT genes from available genomes and from the de novo leaf transcriptome assemblies to generate a phylogenetic tree of the $D C T$ family. The resulting phylogeny shows that DCT4 transcripts form a distinct subclade from the DCT1 clade (Figure 3). The absence of DCT4 transcript expression does not rule out the existence of the gene in the genome. We also used polymerase chain reaction (PCR) to survey for DCT genes in the genomes of grass species for which whole-genome assemblies were not available. We designed conserved primers (non-degenerate or minimally degenerate) to small regions unique to each of the three $D C T$ genes using PrimaClade (Gadberry et al., 2005). We detected DCT1 and DCT2 in the genomes of all species tested (Table 1, 
Supplemental Figure 1). DCT4, however, was detected only in the genomes of NADP-ME $\mathrm{C}_{4}$ species of the PACMAD clade, excluding Z. mays (Table 1, Supplemental Figure 2).

\section{Expression of malate transporter genes in NADP-ME $\mathrm{C}_{4}$ grasses}

$\mathrm{C}_{3}$ species and U. fusca, a PEPCK $\mathrm{C}_{4}$ species, express both DCT1 and DCT2 at low levels in leaves (Table 1 ). $\mathrm{C}_{4}$ NADP-ME species of the PACMAD clade generally express one DCT gene in leaves at a high level and also express one or two other DCT genes at low levels (Table 1). We did not find an apparent lineagespecific pattern for the expression of the predominant DCT gene in the NADP-ME species we analyzed. This finding is consistent with random evolutionary processes underlying the sub-functionalization of members of the $D C T$ family. Interestingly, Z. mays is the only species we examined in which $D C T 2$ is the predominantly expressed $D C T$ gene (Table 1). We also examined the expression of the non-DCT malate transporter OMT1 gene in the leaves of grasses (Table 1). Interestingly, we found that while OMT1 expression was generally abundant, there was no consistent pattern of relative expression between the $D C T$ and $O M T$ genes within the NADP-ME $\mathrm{C}_{4}$ species (Table 1).

\section{DISCUSSION}

\section{Evolution of the $D C T$ gene family in grasses}

We identified DCT4 as a new member of the DCT gene family in the grasses (Figure 1). Our analysis suggests that DCT4 is present in some $C_{4}$ NADP-ME PACMAD grasses. DCT1 and DCT2 appear to have originated from a duplication of a single DCT gene after the monocot-eudicot split (Taniguchi et al., 2004) and DCT4 arose from a duplication of DCT1 at the root of the PACMAD grasses (Figure 3). The expression of $D C T$ genes in the grasses that we analyzed exhibited no clear lineage-specific patterns (Table 1). Therefore, we propose that different members of the DCT family subfunctionalized for photosynthetic malate transport in the BS cells of $\mathrm{C}_{4}$ grasses of the PACMAD clade.

This work also confirms the importance of including syntenic and expression data in assigning orthology across species, and of developing multiple models for $\mathrm{C}_{4}$ photosynthesis in the grasses. SiDCT4 was previously mis-annotated as the ortholog of DCT2 (John et al., 2014), likely because of the lower expression level of SiDCT2 (Seita.9G375100) in leaf tissue. The use of different malate transporters, for example, DCT4 in S. bicolor and S. italica, or DCT2 in Z. mays, suggests that multiple evolutionary paths resulted in the development of an active $C_{4}$ NADP-ME photosynthetic cycle. It is interesting to note 
common origins of $\mathrm{C}_{4}$ photosynthesis are often defined based on the predominant decarboxylase utilized, thus maize and sorghum are considered to have evolved from a common $\mathrm{C}_{4}$ ancestor. This analysis suggests that rather than being static, biochemical adaptations continued after the divergence of maize and sorghum lineages. Thus, optimizations of $\mathrm{C}_{4}$ activities may be continuous as breeding pressures or climate change alters ecological niches of individual species.

\section{Various $\mathrm{C}_{4}$ subtype combinations have different transport requirements}

The variation of expression levels among the different malate transporters within each NADP-ME species (Table 1) suggests different transport requirements during $\mathrm{C}_{4}$ photosynthesis. This supposition is in agreement with the view that the three subtypes of $C_{4}$ photosynthesis are mixed rather than exclusive (Hatch, 1971; Chapman and Hatch, 1979; Furbank, 2011; Pick et al., 2011; Wang et al., 2014b). For example, Z. mays utilizes both the NADP-ME (75\%) and PEPCK (25\%) pathways to fix carbon (Chapman and Hatch, 1979; Wingler et al., 1999; Weissmann et al., 2015), and has similar expression levels of DCT2 and OMT1 and low expression of DCT1. S. bicolor moves carbon through both malate and aspartate, although no PEPCK activity was detected in its leaves (Chapman and Hatch, 1979). S. bicolor has similar expression levels for DCT4 and DCT1 and high expression of OMT1 (Table 1). Other grass species may have dicarboxylate transporter expression ratios that correspond to their unique combination of $\mathrm{C}_{4}$ subsystems. For example, OMT1 is highly expressed in U. fusca, $\sim 3-7$ fold higher than DCT2 or DCT1, respectively. OMT1 transports dicarboxylates, excluding those containing an amino group (Taniguchi et al., 2002; Taniguchi et al., 2004). Thus, in PEPCK $C_{4}$ plants, OMT1 may move oxaloacetate into the mesophyll chloroplast, and 2-oxoglutarate out, to support the high production of aspartate needed to maintain the photosynthetic cycle (Rathnam and Edwards, 1977). Interestingly, both ZmOMT1 and SbOMT1 are only slightly differentially expressed in the M cells (Figure 2). As the loss of DCT2 in Z. mays prevents movement of malate into the BS chloroplast (Weissmann et al., 2015), OMT1 cannot be moving malate into the BS chloroplast alongside DCT2. But OMT1 may also have a role in organic acid metabolism in both cell types, such as shuttling reducing equivalents in organelles other than the chloroplast (Pleite et al., 2005).

\section{CONCLUSIONS}

Our results show that the newly identified member of the $D C T$ gene family, SbDCT4, is an efficient malate transporter. Based on the expression patterns of malate transporters among the grasses, we suggest that different members of the $D C T$ family may have evolved multiple roles in $\mathrm{C}_{4}$ photosynthesis. 
Further studies will be needed to verify the subcellular localization of these proteins and to define their specific metabolic functions. Characterizing the various combinations of $\mathrm{C}_{4}$ photosynthetic subsystems in grasses will facilitate the exploitation of DCT genes, through breeding or engineering, to improve the performance of crop plants and increase yield.

\section{MATERIALS AND METHODS}

\section{Identification of DCT4 genes in Sorghum and other grasses}

We used QUOTA-ALIGN (Tang et al., 2011) to identify syntenic orthologous regions in grass species with sequenced genomes, following the protocol described in (Zhang et al., 2017) . To find homologous genes at non-syntenic locations, we used two complementary approaches. For species with sequenced genomes, we used LASTZ (Harris, 2007) to align the coding sequence of the primary transcript annotated in Phytozome (https://phytozome.jgi.doe.gov) to the genome assembly. For species without assembled genomes, we used LASTZ to align the coding sequence of the primary transcript from Phytozome to transcript assemblies generated by Trinity (Grabherr et al., 2011).

\section{Measurements of malate transport}

We cloned each of the three SBDCT CDNAs between the promoter and terminator of yeast GAL2 in the pTV3e vector (Nishizawa et al., 1995). We transformed the plasmids into yeast LBY416 cells and selected transformants on tryptophan-deficient agar plates. We prepared a crude membrane fraction from the selected yeast transformants. We used a freeze-thaw technique to reconstitute liposomes for the measurement of the uptake of $\left[{ }^{14} \mathrm{C}\right]$ malate (Taniguchi et al., 2002).

\section{Phylogenetic analysis of $D C T$ homologs}

DCT coding sequences for Z. mays, S. bicolor, S. italica, B. distachyon, O. sativa, D. oligosanthes, and U. fusca were from Phytozome (https://phytozome.jgi.doe.gov). We used BLASTN (Altschul et al., 1990) to search de novo assembled leaf transcriptomes (Huang, manuscript in preparation) from the $\mathrm{C}_{4}$ grass species A. congesta, E. aristidea, C. laxum, D. dinteri, A. pubensis, E. esculenta, P. vaginatum, and A. hirta with the DCT sequences from maize, Setaria, and Sorghum as queries. We used ProGraphMSA to generate a codon-based sequence alignment (Szalkowski, 2012). We used MEGA6 (Tamura et al., 2013), with default parameters and the branch support values based on 1,000 bootstraps, to generate the phylogenetic reconstruction with the maximum likelihood method and based on the nucleotides in the third position of codons (Simmons et al., 2006). 


\section{Analysis of gene expression for decarboxylase transporters in grasses}

For species with published leaf transcriptome profiles (Ouyang et al., 2007; Li et al., 2010; Zhang et al., 2012; Schnable, 2014; Wang et al., 2014a; Studer et al., 2016), gene expression levels were calculated and normalized, for each species, as Transcripts Per Million (TPM). For the other species, the normalized TPM values were based on de novo transcriptome assemblies (Huang et al., manuscript in preparation). The values in Table 1 only allow for intraspecies comparisons among the decarboxylase transporters.

\section{Identification of DCT4 in species without sequenced genomes}

We aligned the coding sequences from each of the DCT genes from Z. mays, S. bicolor, S. italica, U. fusca, B. distachyon, O. sativa, D. oligosanthes, A. congesta, E. aristidea, C. laxum, D. dinteri, A. pubensis, E. esculenta, P. vaginatum, and A. hirta using PAL2NAL (Suyama et al., 2006). The resulting multiple sequence alignment enabled the design of non-degenerate or minimally degenerate PCR primers (Table 3) using PrimaClade (Gadberry et al., 2005).

Jacob D. Washburn and J. Chris Pires (University of Missouri, Columbia) kindly provided genomic DNA from A. congesta, E. aristidea, D. dinteri, A. pubensis, E. esculenta, and A. hirta (Washburn et al., 2015). We used a CTAB-based method to extract genomic DNA from C. laxum, P. vaginatum, Z. mays, S. bicolor, S. italica, and B. distachyon (Weissmann et al., 2015). Z. mays and B. distachyon were the negative controls for DCT4 and the positive controls for DCT1 and DCT2. S. bicolor and S. italica were the positive controls for DCT1, DCT2, and DCT4.

We conducted amplification of DCT genes by PCR using a $25-\mu \mathrm{l}$ reaction mix and an ABI 2720 Thermal cycler. The reaction mixture included $2.5 \mu \mathrm{l}$ of $10 \mathrm{X}$ Buffer, $2.5 \mu \mathrm{l}$ of $10 \mu \mathrm{M}$ solutions of forward and reverse primers, $2 \mu \mathrm{l}$ of $2.5 \mathrm{mM}$ dNTP stock, $14 \mu \mathrm{l}$ of nuclease-free water, $0.5 \mu \mathrm{l}$ of Choice Taq enzyme, and $1 \mu \mathrm{l}$ of $100 \mathrm{ng} / \mu \mathrm{l}$ DNA. We performed PCR reactions as described in Table 3 with $5 \mu \mathrm{l}$ of loading dye added to each reaction. Aliquots of $13 \mu \mathrm{l}$ were loaded on 3\% agarose gels (Invitrogen UltraPure Agarose 1000, 1X TAE buffer, Invitrogen SYBR Safe Gel Stain) and electrophoresed for 30 minutes at 100 volts. We based size estimates on $100 \mathrm{bp}$ and 50 bp DNA markers (GoldBio). 
Table 1. Genomic presence or absence and whole leaf TPM values for dicarboxylate transporter genes in grass leaves ${ }^{1}$.

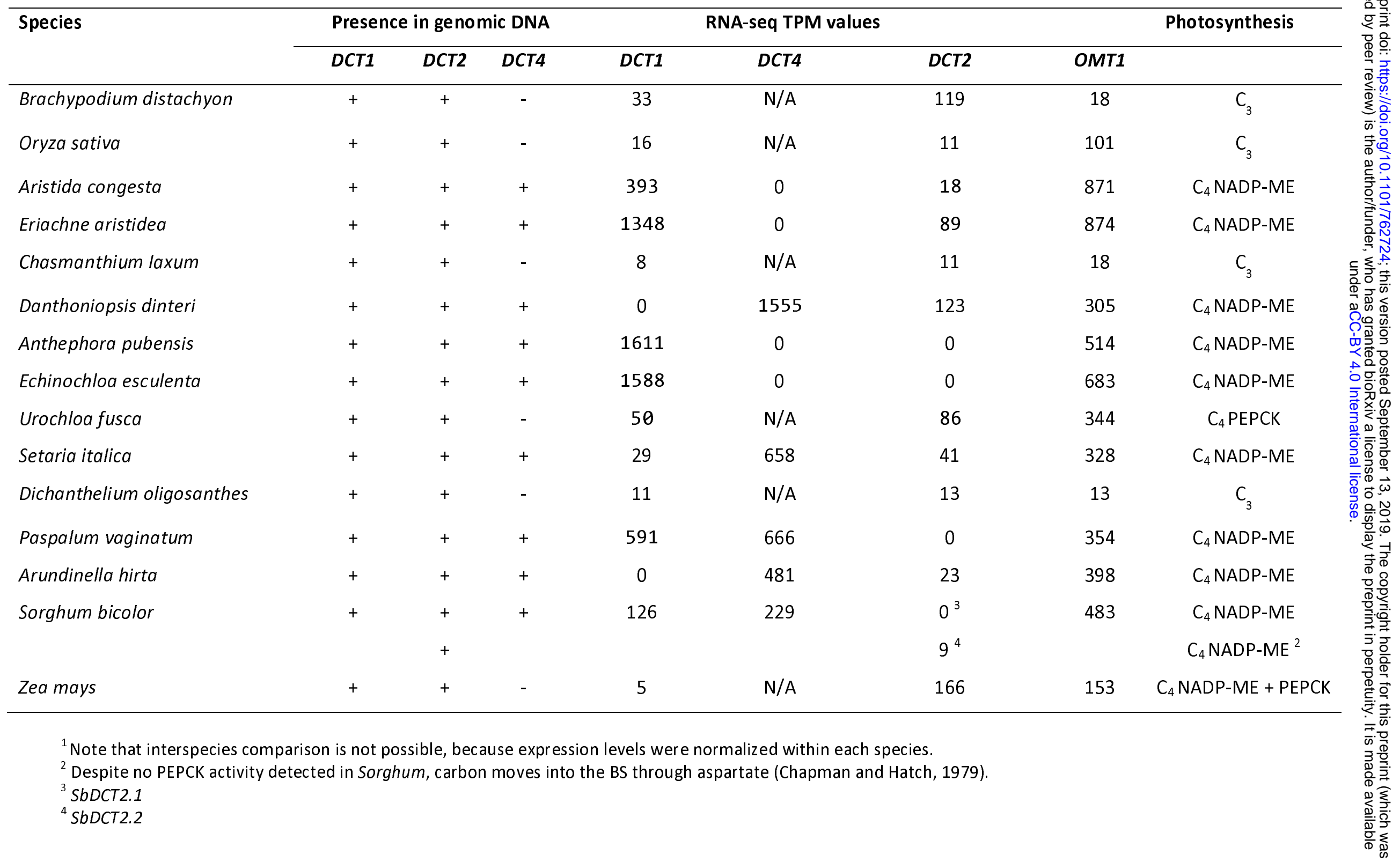


bioRxiv preprint doi: https://doi.org/10.1101/762724; this version posted September 13, 2019. The copyright holder for this preprint (which was not certified by peer review) is the author/funder, who has granted bioRxiv a license to display the preprint in perpetuity. It is made available under aCC-BY 4.0 International license.

Table 2. $K_{\mathrm{m}}$ of malate for recombinant DCT proteins ${ }^{1}$ demonstrates the ability of SbDCT4 to transport malate efficiently.

\begin{tabular}{llll}
\hline \multicolumn{4}{c}{$\boldsymbol{K}_{\mathbf{m}}(\mathrm{mM})$} \\
\hline S. bicolor & DCT1 & DCT2 & DCT4 \\
${\text { Z. } \text { mays }^{2}}^{2}$ & $1.24 \pm 0.14$ & $0.71 \pm 0.10$ & $1.13 \pm 0.10$ \\
\hline
\end{tabular}

${ }^{1}$ The values are the means of three independent experiments \pm SE.

${ }^{2}$ Kinetic values from a previous report (Taniguchi et al., 2004). 
Table 3. Primers and PCR conditions for the amplification of grass DCT genes.

\begin{tabular}{|c|c|c|c|}
\hline Primer Pair & Forward $5^{\prime}-3^{\prime}{ }^{1}$ & Reverse $5^{\prime}-3^{\prime}{ }^{1}$ & Cycling Conditions \\
\hline \multirow[t]{3}{*}{ DCT1 } & CACCAACGAGGTCATCTGG & AGTAGGTGGCGATDCGGTC & $94^{\circ} \mathrm{C} 3 \mathrm{~min},\left[94^{\circ} \mathrm{C} 45 \mathrm{sec}\right.$ \\
\hline & & & $\left.58^{\circ} \mathrm{C} 30 \mathrm{sec}, 72^{\circ} \mathrm{C} 1 \mathrm{~min}\right] \mathrm{x}$ \\
\hline & & & $30,72^{\circ} \mathrm{C} 10 \mathrm{~min}, 4^{\circ} \mathrm{C} \infty$ \\
\hline \multirow[t]{6}{*}{ DCT2 } & CVTGGATGTCRAATTGTGTTG & TGGCTTGCAAABADATAGTGAA & $94^{\circ} \mathrm{C} 3 \mathrm{~min},\left[94^{\circ} \mathrm{C} 45 \mathrm{sec}\right.$ \\
\hline & & & $58^{\circ} \mathrm{C}-52^{\circ} \mathrm{C}\left(-0.5^{\circ} \mathrm{C} /\right.$ cycle $)$ \\
\hline & & & $\left.30 \mathrm{sec}, 72^{\circ} \mathrm{C} 1 \mathrm{~min}\right] \times 14$ \\
\hline & & & {$\left[94^{\circ} \mathrm{C} 45 \mathrm{sec}, 52^{\circ} \mathrm{C} 30 \mathrm{sec}\right.$} \\
\hline & & & $\left.72^{\circ} \mathrm{C} 1 \mathrm{~min}\right] \times 16,72^{\circ} \mathrm{C} 10$ \\
\hline & & & $\min , 4^{\circ} \mathrm{C} \infty$ \\
\hline \multirow[t]{3}{*}{ DCT4 } & CTTYGTCAAGTGGCTCGG & GACTTGATGATSGGCAGGA & $94^{\circ} \mathrm{C} 3 \mathrm{~min},\left[94^{\circ} \mathrm{C} 45 \mathrm{sec}\right.$ \\
\hline & & & $\left.60^{\circ} \mathrm{C} 30 \mathrm{sec}, 72^{\circ} \mathrm{C} 1 \mathrm{~min}\right] \mathrm{x}$ \\
\hline & & & $30,72^{\circ} \mathrm{C} 10.0 \mathrm{~min}, 4^{\circ} \mathrm{C} \infty$. \\
\hline
\end{tabular}

${ }^{1} B=C+G+T, D=A+G+T, R=A+G, S=C+G, V=A+C+G, Y=C+T$. 


\section{FIGURE LEGENDS}

Figure 1. CoGe (https://genomevolution.org/coge/) genome viewer screenshots depicting the conservation and genomic contexts of DCT genes in Z. mays and S. bicolor. Colored lines between panels show conserved genes. SBDCT4 shows high sequence conservation with other DCT genes, but is not a syntenic ortholog of $Z m D C T 2$, as shown by the lack of conservation in neighboring genes.

Figure 2. Differential expression of malate transporters in Z. mays, and S. bicolor leaves between the BS and $\mathrm{M}$ cells. The genome of Z. mays has one copy of DCT2 and does not contain DCT4, and ZmDCT2 is highly enriched in BS cells. Sorghum bicolor has two copies of DCT2 (DCT2.1, and DCT2.2) in the syntenic genomic location that are the result of gene duplication. S. bicolor also expresses DCT4, which is highly enriched in BS cells. Both species express OMT1 and DCT1, which are only slightly enriched in the M cells. Red bars represent enrichment in the BS cells. Blue bars represent enrichment in the $M$ cells. The white numbers inside the bars represent the significance ( $p$-value) of the log2(FoldChange).

Figure 3. A phylogenetic tree of the DCT family in the grasses showing that DCT4 is a subclade of DCT1. The DCT1, DCT2, and DCT4 gene lineages are black, blue, and red, respectively. The length of the branches represents the evolutionary distance between ancestor to descendent nodes. The numbers represent the confidence level of the specific branch.

Supplemental Figure 1. Gel image showing that DCT1 and DCT2 genes are present in all grass species tested.

Supplemental Figure 2. Gel image showing the presence or absence of DCT4 genes from species lacking genome assemblies. Negative controls were $Z$. mays and B. distachyon, and positive controls were $S$. bicolor and S. italica. 


\section{REFERENCES}

Altschul, S.F., Gish, W., Miller, W., Myers, E.W., and Lipman, D.J. (1990). Basic local alignment search tool. J Mol Biol 215, 403-410.

Bennetzen, J.L. (2000). Comparative sequence analysis of plant nuclear genomes:m microcolinearity and its many exceptions. Plant Cell 12, 1021-1029.

Bennetzen, J.L., and Freeling, M. (1997). The unified grass genome: synergy in synteny. Genome research 7, 301-306.

Brautigam, A., Hoffmann-Benning, S., and Weber, A.P. (2008). Comparative proteomics of chloroplast envelopes from $\mathrm{C} 3$ and $\mathrm{C} 4$ plants reveals specific adaptations of the plastid envelope to $\mathrm{C} 4$ photosynthesis and candidate proteins required for maintaining $\mathrm{C} 4$ metabolite fluxes. Plant Physiol 148, 568-579.

Chapman, K.S., and Hatch, M.D. (1979). Aspartate stimulation of malate decarboxylation in Zea mays bundle sheath cells: possible role in regulation of $\mathrm{C} 4$ photosynthesis. Biochemical and biophysical research communications $\mathbf{8 6}, 1274-1280$.

Chen, M., SanMiguel, P., de Oliveira, A.C., Woo, S.S., Zhang, H., Wing, R.A., and Bennetzen, J.L. (1997). Microcolinearity in sh2-homologous regions of the maize, rice, and sorghum genomes. Proceedings of the National Academy of Sciences of the United States of America 94, 34313435.

Davidson, R.M., Gowda, M., Moghe, G., Lin, H., Vaillancourt, B., Shiu, S.H., Jiang, N., and Robin Buell, C. (2012). Comparative transcriptomics of three Poaceae species reveals patterns of gene expression evolution. The Plant journal : for cell and molecular biology 71, 492-502.

Ding, Z., Weissmann, S., Wang, M., Du, B., Huang, L., Wang, L., Tu, X., Zhong, S., Myers, C., Brutnell, T.P., Sun, Q., and Li, P. (2015). Identification of Photosynthesis-Associated C4 Candidate Genes through Comparative Leaf Gradient Transcriptome in Multiple Lineages of C3 and C4 Species. PloS one 10, e0140629.

Furbank, R.T. (2011). Evolution of the C(4) photosynthetic mechanism: are there really three $C(4)$ acid decarboxylation types? Journal of experimental botany 62, 3103-3108.

Gadberry, M.D., Malcomber, S.T., Doust, A.N., and Kellogg, E.A. (2005). Primaclade--a flexible tool to find conserved PCR primers across multiple species. Bioinformatics 21, 1263-1264.

Grabherr, M.G., Haas, B.J., Yassour, M., Levin, J.Z., Thompson, D.A., Amit, I., Adicon is, X., Fan, L., Raychowdhury, R., Zeng, Q., Chen, Z., Mauceli, E., Hacohen, N., Gnirke, A., Rhind, N., di Palma, F., Birren, B.W., Nusbaum, C., Lindblad-Toh, K., Friedman, N., and Regev, A. (2011). Full-length transcriptome assembly from RNA-Seq data without a reference genome. Nature biotechnology 29, 644-652.

Harris, R.S. (2007). Improved Pairwise Alignmnet of Genomic DNA. In Computer Science and Engineering (Philadelphia: pennsylvania state university).

Hatch, M.D. (1971). The C 4 -pathway of photosynthesis. Evidence for an intermediate pool of carbon dioxide and the identity of the donor C 4 -dicarboxylic acid. The Biochemical journal 125, 425432.

Hatch, M.D., and Slack, C.R. (1966). Photosynthesis by sugar-cane leaves. A new carboxylation reaction and the pathway of sugar formation. The Biochemical journal 101, 103-111.

Huang, P., Studer, A.J., Schnable, J.C., Kellogg, E.A., and Brutnell, T.P. (2017). Cross species selection scans identify components of $\mathrm{C} 4$ photosynthesis in the grasses. Journal of experimental botany 68, 127-135.

Jin, J., Kong, J., Qiu, J., Zhu, H., Peng, Y., and Jiang, H. (2016). High level of microsynteny and purifying selection affect the evolution of WRKY family in Gramineae. Development genes and evolution 226, 15-25. 
John, C.R., Smith-Unna, R.D., Woodfield, H., Covshoff, S., and Hibberd, J.M. (2014). Evolutionary convergence of cell-specific gene expression in independent lineages of $\mathrm{C} 4$ grasses. Plant Physiol $165,62-75$.

Kinoshita, H., Nagasaki, J., Yoshikawa, N., Yamamoto, A., Takito, S., Kawasaki, M., Sugiyama, T., Miyake, H., Weber, A.P.M., and Taniguchi, M. (2011). The chloroplastic 2-oxoglutarate/malate transporter has dual function as the malate valve and in carbon/nitrogen metabolism. Plant Journal 65, 15-26.

Kumar, S., Mohan, A., Balyan, H.S., and Gupta, P.K. (2009). Orthology between genomes of Brachypodium, wheat and rice. BMC research notes 2, 93.

Li, P.H., Ponnala, L., Gandotra, N., Wang, L., Si, Y.Q., Tausta, S.L., Kebrom, T.H., Provart, N., Patel, R., Myers, C.R., Reidel, E.J., Turgeon, R., Liu, P., Sun, Q., Nelson, T., and Brutnell, T.P. (2010). The developmental dynamics of the maize leaf transcriptome. Nature Genetics 42, 1060-U1051.

Linka, N., and Weber, A.P.M. (2010). Intracellular Metabolite Transporters in Plants. Molecular Plant 3, 21-53.

Nishizawa, K., Shimoda, E., and Kasahara, M. (1995). Substrate recognition domain of the Gal2 galactose transporter in yeast Saccharomyces cerevisiae as revealed by chimeric galactoseglucose transporters. The Journal of biological chemistry 270, 2423-2426.

Ouyang, S., Zhu, W., Hamilton, J., Lin, H., Campbell, M., Childs, K., Thibaud-Nissen, F., Malek, R.L., Lee, Y., Zheng, L., Orvis, J., Haas, B., Wortman, J., and Buell, C.R. (2007). The TIGR Rice Genome Annotation Resource: improvements and new features. Nucleic acids research 35, D883-887.

Pick, T.R., Brautigam, A., Schluter, U., Denton, A.K., Colmsee, C., Scholz, U., Fahnenstich, H., Pieruschka, R., Rascher, U., Sonnewald, U., and Weber, A.P.M. (2011). Systems Analysis of a Maize Leaf Developmental Gradient Redefines the Current C-4 Model and Provides Candidates for Regulation. Plant Cell 23, 4208-4220.

Pleite, R., Pike, M.J., Garces, R., Martinez-Force, E., and Rawsthorne, S. (2005). The sources of carbon and reducing power for fatty acid synthesis in the heterotrophic plastids of developing sunflower (Helianthus annuus L.) embryos. Journal of experimental botany 56, 1297-1303.

Rao, X., Lu, N., Li, G., Nakashima, J., Tang, Y., and Dixon, R.A. (2016). Comparative cell-specific transcriptomics reveals differentiation of $\mathrm{C} 4$ photosynthesis pathways in switchgrass and other C4 lineages. Journal of experimental botany 67, 1649-1662.

Rathnam, C.K.M., and Edwards, G.E. (1977). C4 acid decarboxylation and co2 donation to photosynthesis in bundle sheath strands and chloroplasts from species representing 3 groups of c4 plants. Arch. Biochem. Biophys. 182, 1-13.

Sanchez-Ken, J.G., and Clark, L.G. (2010). Phylogeny and a new tribal classification of the Panicoideae s.l. (Poaceae) based on plastid and nuclear sequence data and structural data. American journal of botany $97,1732-1748$.

Schnable, J.C. (2014). Urochloa fusca LBJWC-52 Annotated Standard Draft (Joint Genome Institute).

Simmons, M.P., Zhang, L.B., Webb, C.T., and Reeves, A. (2006). How can third codon positions outperform first and second codon positions in phylogenetic inference? An empirical example from the seed plants. Systematic biology 55, 245-258.

Studer, A.J., Schnable, J.C., Weissmann, S., Kolbe, A.R., McKain, M.R., Shao, Y., Cousins, A.B., Kellogg, E.A., and Brutnell, T.P. (2016). The draft genome of the C3 panicoid grass species Dichanthelium oligosanthes. Genome Biol 17, 223.

Suyama, M., Torrents, D., and Bork, P. (2006). PAL2NAL: robust conversion of protein sequence alignments into the corresponding codon alignments. Nucleic acids research 34, W609-612.

Szalkowski, A.M. (2012). Fast and robust multiple sequence alignment with phylogeny-aware gap placement. BMC bioinformatics 13, 129. 
Tamura, K., Stecher, G., Peterson, D., Filipski, A., and Kumar, S. (2013). MEGA6: Molecular Evolutionary Genetics Analysis version 6.0. Mol Biol Evol 30, 2725-2729.

Tang, H., Lyons, E., Pedersen, B., Schnable, J.C., Paterson, A.H., and Freeling, M. (2011). Screening synteny blocks in pairwise genome comparisons through integer programming. BMC bioinformatics 12, 102.

Taniguchi, M., Taniguchi, Y., Kawasaki, M., Takeda, S., Kato, T., Sato, S., Tahata, S., Miyake, H., and Sugiyama, T. (2002). Identifying and characterizing plastidic 2-oxoglutarate/malate and dicarboxylate transporters in Arabidopsis thaliana. Plant and Cell Physiology 43, 706-717.

Taniguchi, Y., Nagasaki, J., Kawasaki, M., Miyake, H., Sugiyama, T., and Taniguchi, M. (2004). Differentiation of dicarboxylate transporters in mesophyll and bundle sheath chloroplasts of maize. Plant Cell Physiol 45, 187-200.

Tausta, S.L., Li, P., Si, Y., Gandotra, N., Liu, P., Sun, Q., Brutnell, T.P., and Nelson, T. (2014). Developmental dynamics of Kranz cell transcriptional specificity in maize leaf reveals early onset of C4-related processes. Journal of experimental botany 65, 3543-3555.

Tikhonov, A.P., SanMiguel, P.J., Nakajima, Y., Gorenstein, N.M., Bennetzen, J.L., and Avramova, Z. (1999). Colinearity and its exceptions in orthologous adh regions of maize and sorghum. Proceedings of the National Academy of Sciences of the United States of America 96, 74097414.

Wang, L., Czedik-Eysenberg, A., Mertz, R.A., Si, Y., Tohge, T., Nunes-Nesi, A., Arrivault, S., Dedow, L.K., Bryant, D.W., Zhou, W., Xu, J., Weissmann, S., Studer, A., Li, P., Zhang, C., LaRue, T., Shao, Y., Ding, Z., Sun, Q., Patel, R.V., Turgeon, R., Zhu, X., Provart, N.J., Mockler, T.C., Fernie, A.R., Stitt, M., Liu, P., and Brutnell, T.P. (2014a). Comparative analyses of $C(4)$ and $C(3)$ photosynthesis in developing leaves of maize and rice. Nature biotechnology 32, 1158-1165.

Wang, Y., Brautigam, A., Weber, A.P.M., and Zhu, X.G. (2014b). Three distinct biochemical subtypes of C-4 photosynthesis? A modelling analysis. Journal of experimental botany 65, 3567-3578.

Washburn, J.D., Schnable, J.C., Davidse, G., and Pires, J.C. (2015). Phylogeny and photosynthesis of the grass tribe Paniceae. American journal of botany 102, 1493-1505.

Weissmann, S., and Brutnell, T.P. (2012). Engineering C4 photosynthetic regulatory networks. Curr Opin Biotechnol 23, 298-304.

Weissmann, S., Ma, F., Furuyama, K., Gierse, J., Berg, H., Shao, Y., Taniguchi, M., Allen, D.K., and Brutnell, T.P. (2015). Interactions of C4 Subtype Metabolic Activities and Transport in Maize Are Revealed through the Characterization of DCT2 Mutants. Plant Cell 28, 466-484.

Wingler, A., Walker, R.P., Chen, Z.H., and Leegood, R.C. (1999). Phosphoenolpyruvate carboxykinase is involved in the decarboxylation of aspartate in the bundle sheath of maize. Plant Physiol 120, 539-546.

Zhang, G., Liu, X., Quan, Z., Cheng, S., Xu, X., Pan, S., Xie, M., Zeng, P., Yue, Z., Wang, W., Tao, Y., Bian, C., Han, C., Xia, Q., Peng, X., Cao, R., Yang, X., Zhan, D., Hu, J., Zhang, Y., Li, H., Li, H., Li, N., Wang, J., Wang, C., Wang, R., Guo, T., Cai, Y., Liu, C., Xiang, H., Shi, Q., Huang, P., Chen, Q., Li, Y., Wang, J., Zhao, Z., and Wang, J. (2012). Genome sequence of foxtail millet (Setaria italica) provides insights into grass evolution and biofuel potential. Nature biotechnology 30, 549-554.

Zhang, Y., Ngu, D.W., Carvalho, D., Liang, Z., Qiu, Y., Roston, R.L., and Schnable, J.C. (2017). Differentially Regulated Orthologs in Sorghum and the Subgenomes of Maize. Plant Cell 29, 1938-1951. 


\section{Figure 1}

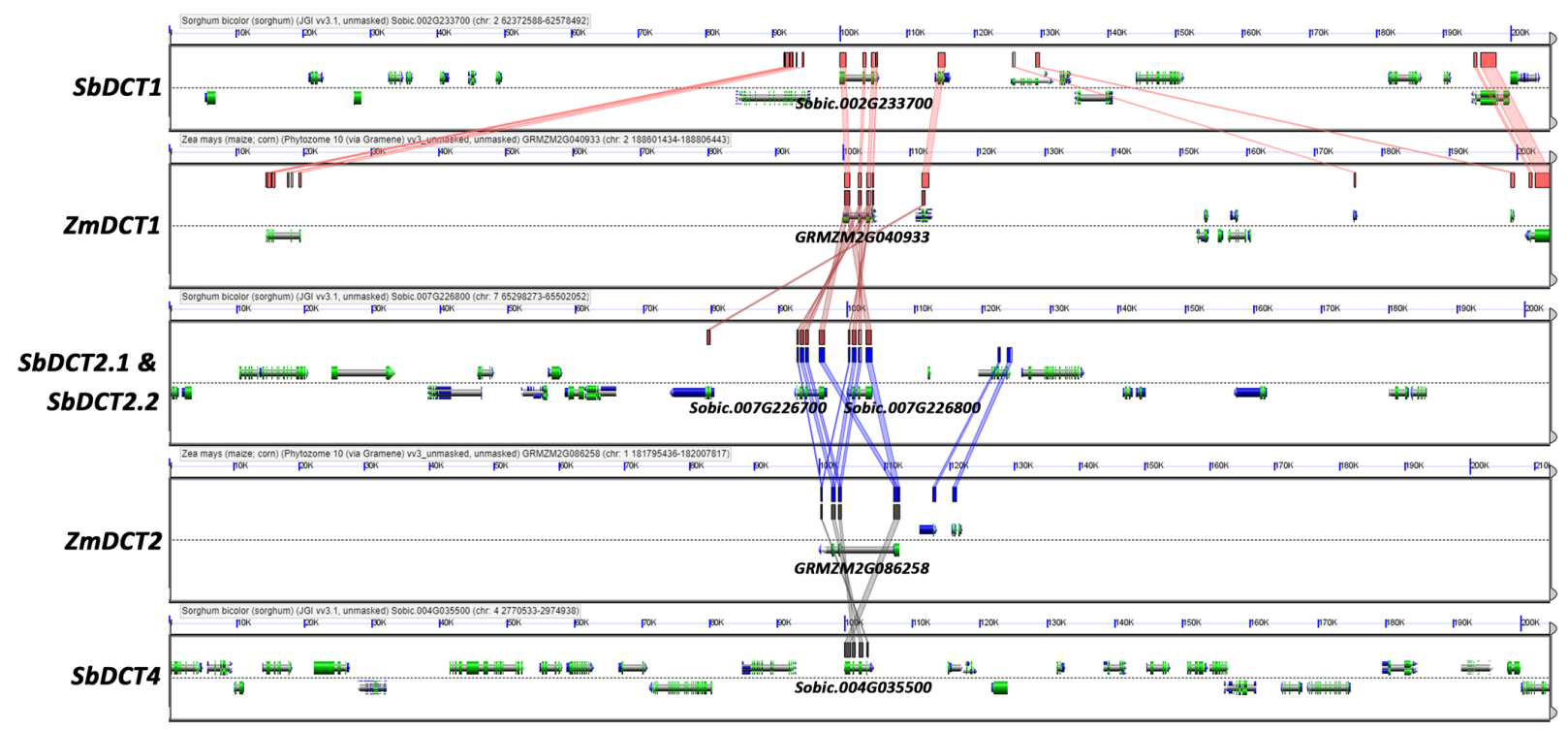


Figure 2

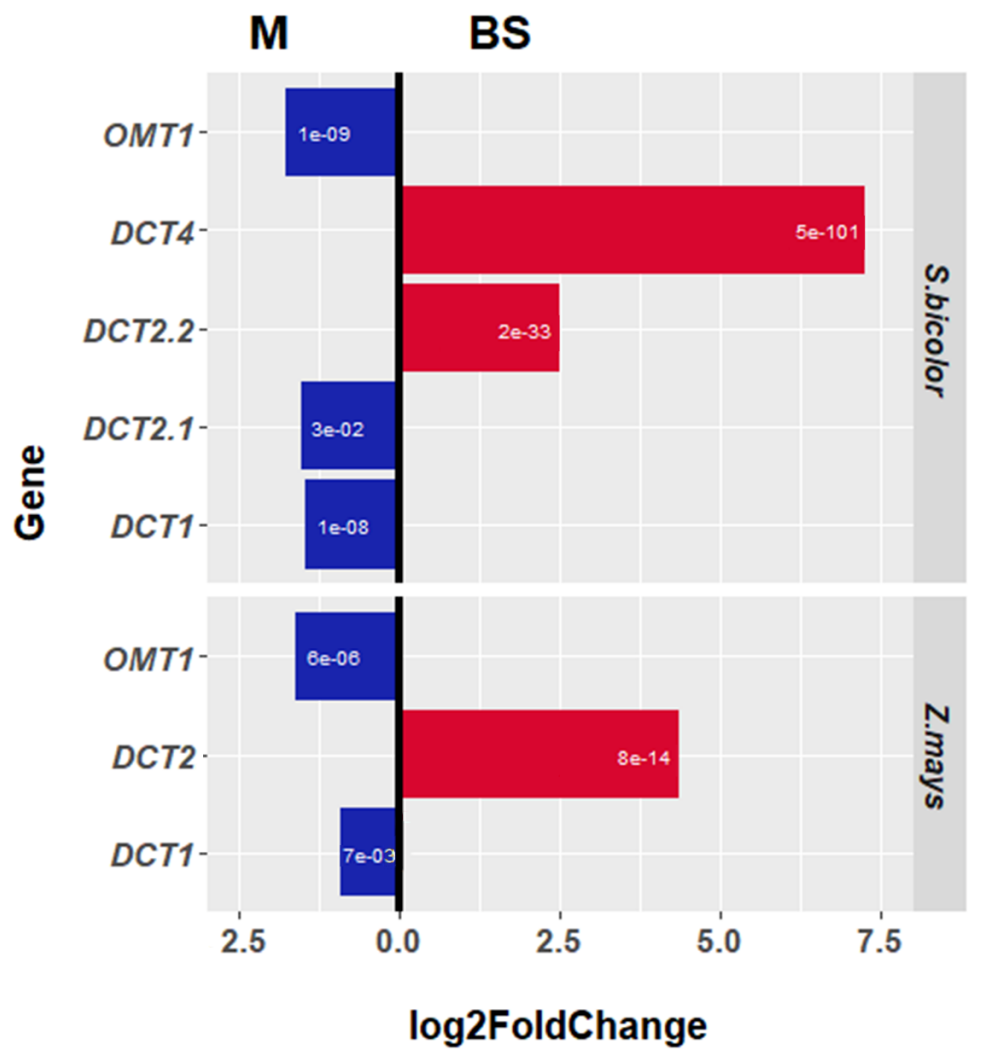


Figure 3

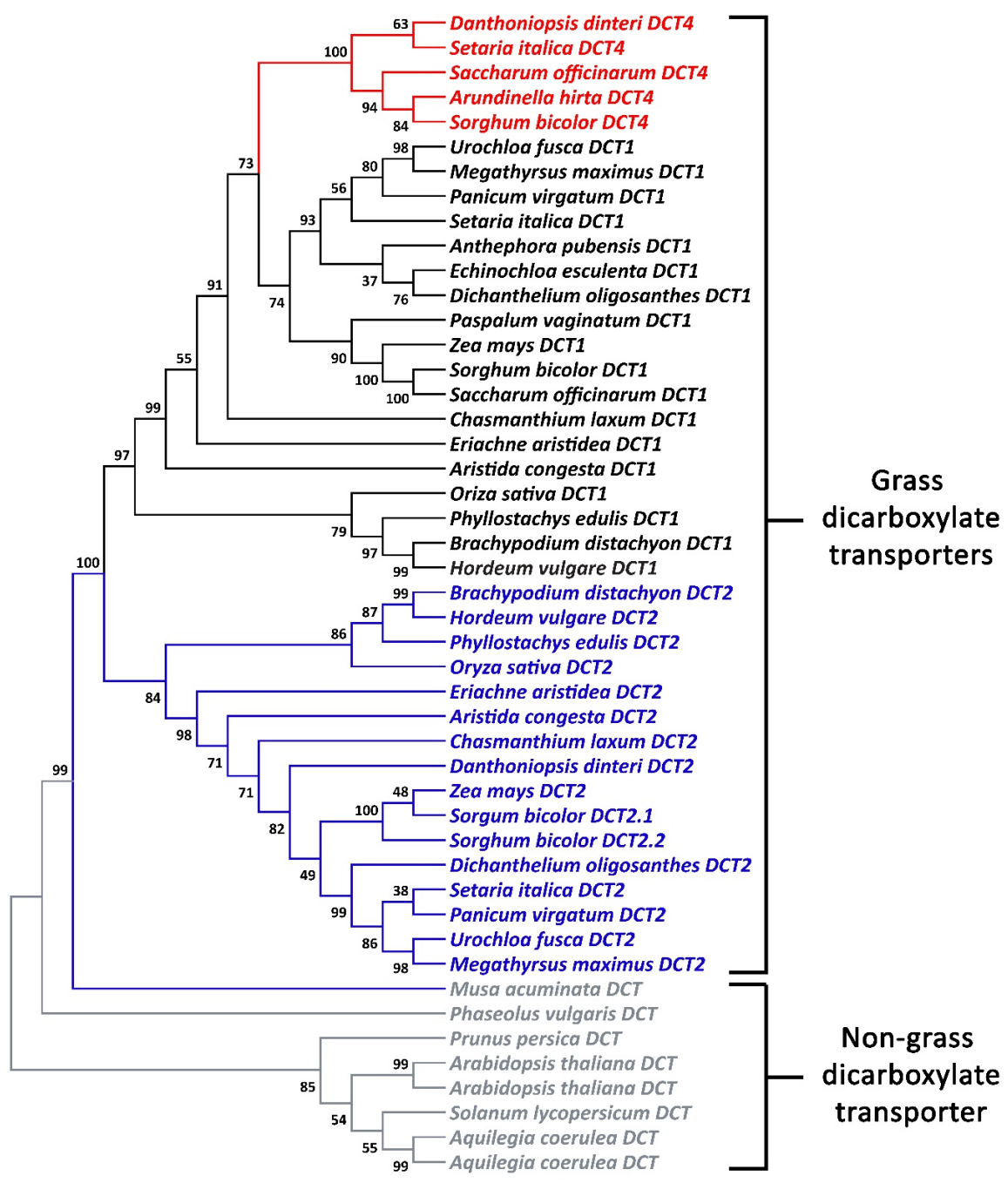




\section{Supplemental Figure 1}

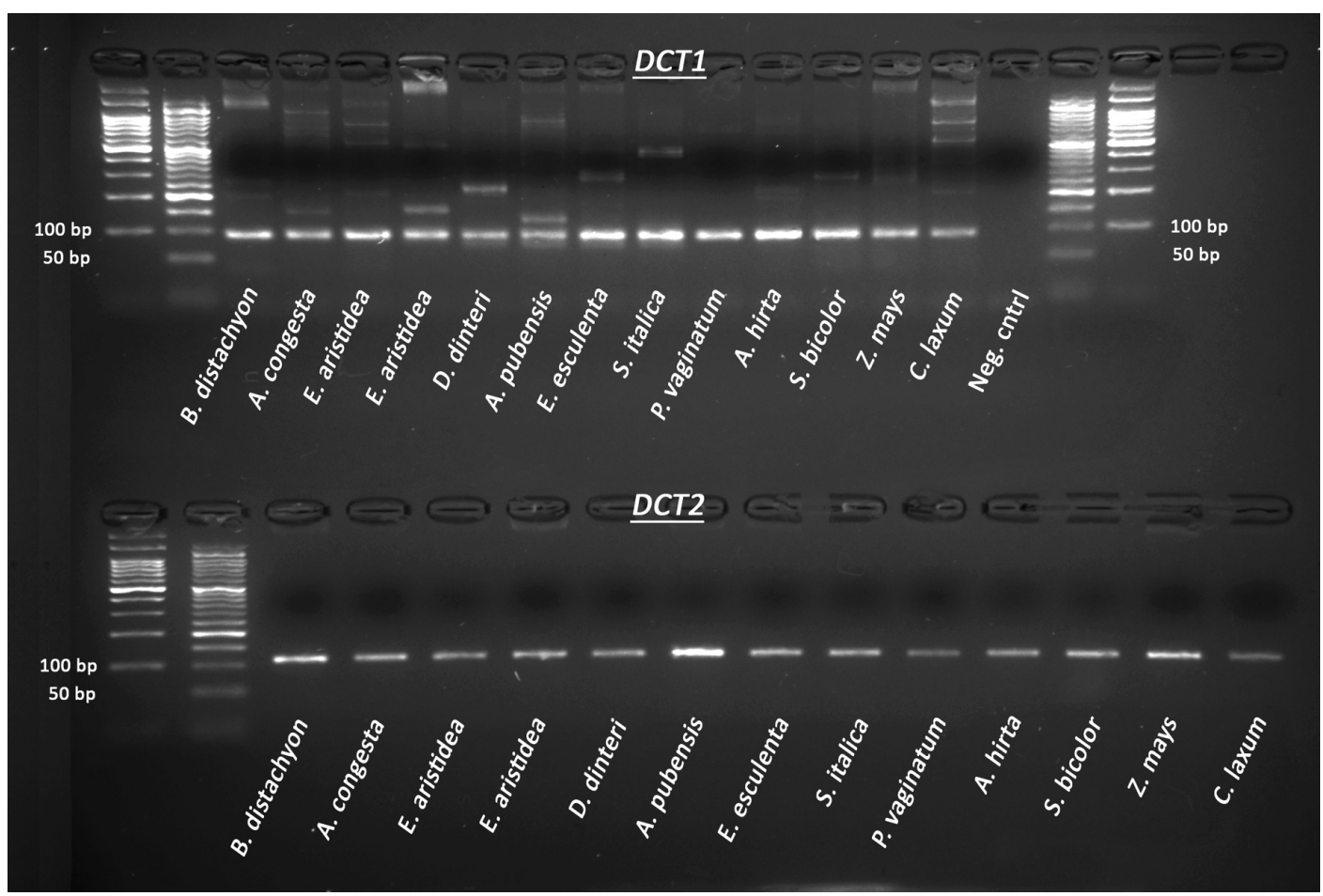




\section{Supplemental Figure 2}

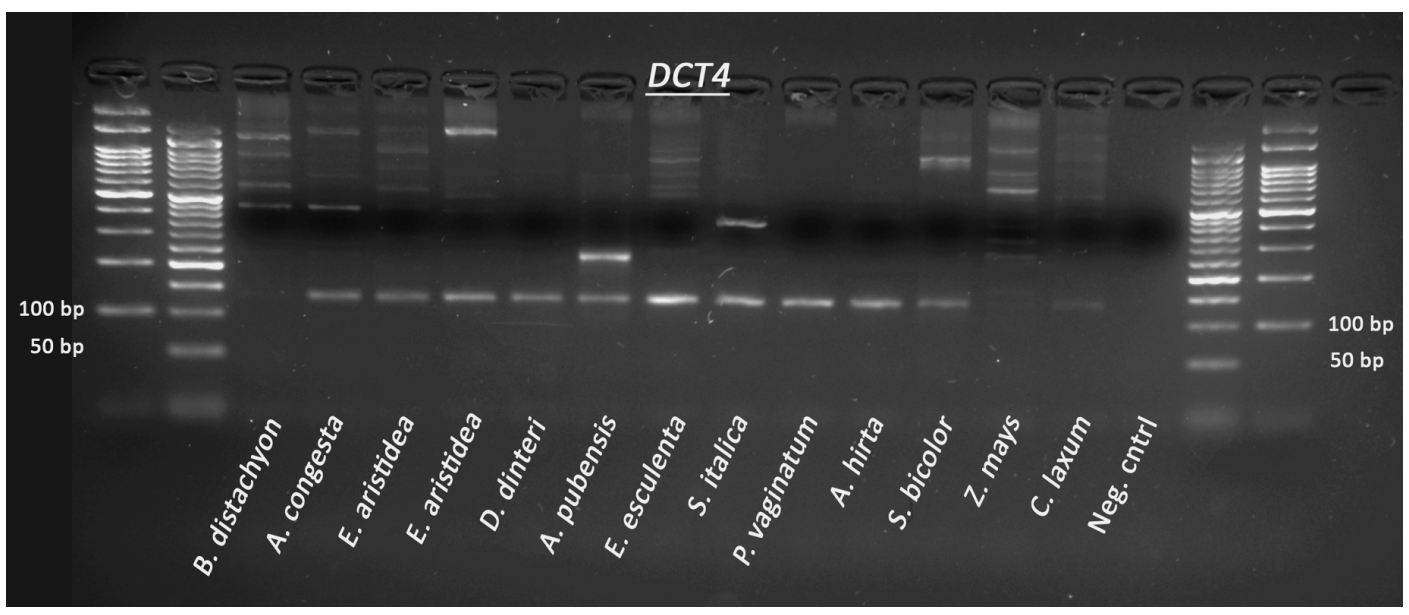

